\title{
THE FAMILY OF ANALYTIC POISSON BRACKETS FOR THE CAMASSA-HOLM HIERARCHY
}

\author{
M. I. GeKhtman ${ }^{\dagger}$ And K. L. VAninsky
}

\begin{abstract}
We consider the integrable Camassa-Holm hierarchy on the line with positive initial data rapidly decaying at infinity. It is known that flows of the hierarchy can be formulated in a Hamiltonian form using two compatible Poisson brackets. In this note we propose a new approach to Hamiltonian theory of the $\mathrm{CH}$ equation. In terms of associated Riemann surface and the Weyl function we write an analytic formula which produces a family of compatible Poisson brackets. The formula includes an entire function $f(z)$ as a parameter. The simplest choice $f(z)=1$ or $f(z)=z$ corresponds to the rational or trigonometric solutions of the Yang-Baxter equation and produces two original Poisson brackets. All other Poisson brackets corresponding to other choices of the function $f(z)$ are new.
\end{abstract}

\section{Introduction.}

1.1. The Camassa-Holm hierarchy. The Camassa-Holm equation, $[3,4]$,

$$
\frac{\partial v}{\partial t}+v \frac{\partial v}{\partial x}+\frac{\partial}{\partial x} R\left[v^{2}+\frac{1}{2}\left(\frac{\partial v}{\partial x}\right)^{2}\right]=0
$$

in which $t \geq 0$ and $-\infty<x<\infty, v=v(x, t)$ is a velocity, and $R$ is the inverse to $L=1-d^{2} / d x^{2}$, i.e.

$$
R[f](x)=\frac{1}{2} \int_{-\infty}^{+\infty} e^{-|x-y|} f(y) d y,
$$

is an approximation to the Euler equation describing an ideal fluid. Introducing the function $m=L[v]$ one writes the equation in the form ${ }^{1}$

$$
m^{\bullet}+(m D+D m) v=0 .
$$

The $\mathrm{CH}$ equation is a Hamiltonian system $m \bullet+\{m, \mathcal{H}\}_{J_{0}}=0$ with the Hamiltonian

$$
\mathcal{H}=\frac{1}{2} \int_{-\infty}^{+\infty} m v d x=\text { energy }
$$

and the bracket

$$
\{A, B\}_{J_{0}}=\int_{-\infty}^{+\infty} \frac{\delta A}{\delta m} J_{0} \frac{\delta B}{\delta m} d x, \quad J_{0}=m D+D m .
$$

Received by the editors August 31, 2007.

$\dagger$ Work supported in part by NSF Grant DMD-0400484.

1 We use notation $D$ for the $x$-derivative and $\bullet$ for the $t$-derivative. We use $\delta$ for the Frechet derivative. 
We consider the $\mathrm{CH}$ equation with nonnegative $(m \geq 0)$ initial data and such decay at infinity that:

$$
\int_{-\infty}^{+\infty} m(x) e^{|x|} d x<\infty
$$

We denote this class of functions by $\mathcal{M}$. For such data a solution of the initial value problem exists for all times, see [7].

The CH Hamiltonian is one of infinitely many conserved integrals of motion

$$
\begin{aligned}
\mathcal{H}_{0} & =\int_{-\infty}^{\infty} \sqrt{m} d x, \\
\mathcal{H}_{1} & =\int_{-\infty}^{\infty} v d x \\
\mathcal{H}_{2} & =\frac{1}{2} \int_{-\infty}^{\infty} v^{2}+(D v)^{2} d x, \\
\mathcal{H}_{3} & =2 \int_{-\infty}^{\infty} v\left[v^{2}+(D v)^{2}\right] d x \\
\mathcal{H}_{4} & =\frac{1}{2} \int_{-\infty}^{\infty} v^{4} d x+\int_{-\infty}^{\infty} v^{2}(D v)^{2} d x+2 \int_{-\infty}^{\infty}\left[v^{2}+\frac{(D v)^{2}}{2}\right] G\left[v^{2}+\frac{(D v)^{2}}{2}\right] d x, \text { etc. }
\end{aligned}
$$

The quantity $\mathcal{H}_{0}$ is a Casimir of the bracket. The integral

$$
\mathcal{H}_{1}=\int_{-\infty}^{+\infty} m d x=\text { momentum }
$$

produces the flow of translation

$$
m^{\bullet}+\left\{m, \mathcal{H}_{1}\right\}_{J_{0}}=m^{\bullet}+D m=0 .
$$

The second is the $\mathrm{CH}$ Hamiltonian $\mathcal{H}_{2}=\mathcal{H}$. The integrals $\mathcal{H}_{3}, \mathcal{H}_{4}$, etc., produce higher flows of the $\mathrm{CH}$ hierarchy.

Let us define the second Poisson bracket

$$
\{A, B\}_{J_{1}}=\int_{-\infty}^{+\infty} \frac{\delta A}{\delta m} J_{1} \frac{\delta B}{\delta m} d x, \quad J_{1}=D-D^{3} .
$$

The conserved quantity $\mathcal{H}_{1}$ is a Casimir of this bracket. The Hamiltonians of the $\mathrm{CH}$ hierarchy satisfy the reccurence relation

$$
J_{0} \frac{\delta \mathcal{H}_{n}}{\delta m}=J_{1} \frac{\delta \mathcal{H}_{n+1}}{\delta m}, \quad n \geq 0 .
$$

For integration of the $\mathrm{CH}$ equation we consider an auxiliary string spectral problem, 2

$$
f^{\prime \prime}(\xi)+\lambda g(\xi) f(\xi)=0, \quad-2 \leq \xi \leq 2 .
$$

The background information for this spectral problem can be found in $[8,11,14]$. The variables $\xi$ and $x$ are related by

$$
x \longrightarrow \xi=2 \tanh \frac{x}{2} \text {. }
$$

\footnotetext{
2 We use prime ' to denote $\xi$-derivative.
} 
Also the potential $g(\xi)$ is related to $m(x)$ by the formula $g(\xi)=m(x) \cosh ^{4} \frac{x}{2}$. For initial data from $\mathcal{M}$ the total mass of associated string is finite : $\int_{-2}^{+2} g(\xi) d \xi<\infty$.

Two important solutions $\varphi(\xi, \lambda)$ and $\psi(\xi, \lambda)$ of the string spectral problem are specified by initial data

$$
\begin{aligned}
\varphi(-2, \lambda) & =1 & \psi(-2, \lambda) & =0 \\
\varphi^{\prime}(-2, \lambda) & =0 & \psi^{\prime}(-2, \lambda) & =1 .
\end{aligned}
$$

The Weyl function, [21], is defined by the formula

$$
E_{0}(\lambda)=-\frac{\varphi(2, \lambda)}{\psi(2, \lambda)}
$$

The Riemann surface $\Gamma$ associated with the string spectral problem consists of two components, $\Gamma_{+}$and $\Gamma_{-}$, which are two copies of the Riemann sphere. The points $\lambda$ 's where two spheres are glued to each other are points of the Dirichlet spectrum. The points $\gamma$ 's on $\Gamma_{-}$are the points of the Newmann spectrum, see Figure 1.

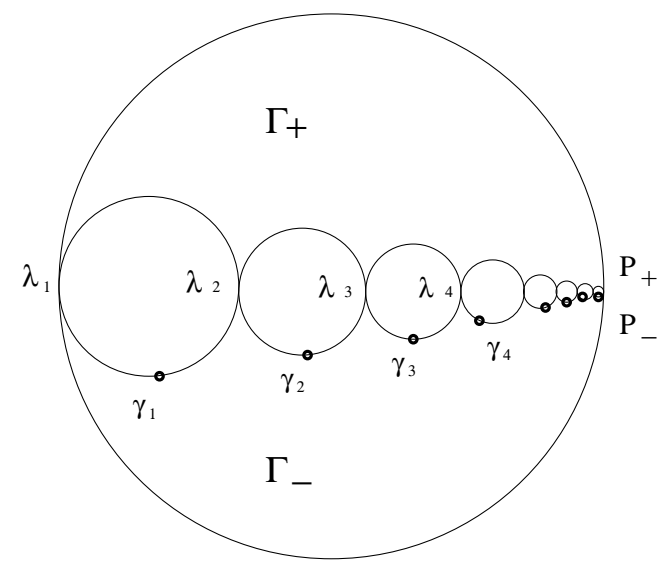

Figure 1. The Riemann surface.

The pair $\left(\Gamma, E_{0}\right)$ provides a parametrization of the phase space $\mathcal{M}$, see [19].

Now we introduce a family of compatible Poisson brackets. Changing spectral variable $\lambda \rightarrow z=-1 / \lambda$ we have

$$
E_{0}(z)=-\frac{1}{4}+\sum_{m=1}^{\infty} \frac{\rho_{m}^{\prime}}{z_{m}-z},
$$

where poles $z_{m}$ accumulate near the origin. Consider a differential $\omega_{p q}^{f}$ on the lower component of the spectral curve $\Gamma_{-}$which depends on the entire function $f(z)$ and two points $p$ and $q$

$$
\omega_{p q}^{f}=\frac{1}{2 \pi i} \frac{f(z) d z}{(z-p)(z-q)} \times E_{0}(z)\left(E_{0}(p)-E_{0}(q)\right) .
$$

Any point $p \in \Gamma_{-}$can be viewed as a function on pairs, $p:\left(\Gamma, E_{0}\right) \rightarrow E_{0}(p)$. For any two points $p$ and $q$ that are away from the poles of some $E_{0}$ we define the Poisson 
bracket in vicinity of the pair $\left(\Gamma, E_{0}\right)$ by the formula

$$
\left\{E_{0}(p), E_{0}(q)\right\}_{\omega^{f}} \equiv \sum_{m} \int_{\widetilde{O}_{m}} \omega_{p q}^{f},
$$

where the circles $O_{m}$ enclose points $z_{m}$ and are traversed clockwise. The map $\left(\Gamma, E_{0}\right) \longrightarrow \mathcal{M}$ induces a Poisson structure on $\mathcal{M}$.

It turns out that if $f(z)=1$, then the Poisson bracket defined by this formula coincides with the bracket (1.2). When $f(z)=z$ the Poisson bracket coincides with (1.4). All other brackets corresponding to other choices of $f(z)$ are new, though we do not know their explicit form on the phase space $\mathcal{M}$.

The formula for $\omega_{p q}^{f}$ has a clear geometrical meaning. The first piece is a differential of the third kind with poles at the points $p$ and $q$. It also can have a pole at infinity depending on the rate of growth of the function $f(z)$. The second piece is quadratic in the function $E_{0}$ and skew-symmetric in $p$ and $q$. The formula has an immediate generalization to higher genus curves, which we will discuss in future publications.

1.2. Historical remarks. V.I. Arnold established a Hamiltonian nature of Euler equations of hydrodynamics using infinite dimensional diffemorphism group, see [1]. Later his Lie group approach was employed by Khesin and Misiolek [16] for the KdV, $\mathrm{CH}$ and HS equations. They considered bi-Hamiltonian structures associated with Euler's equations generated by a certain two-parameter family of metrics on the Virasoro group and have shown that the $\mathrm{KdV}, \mathrm{CH}$ and $\mathrm{HS}$ equations are bi-Hamiltonian systems on the dual of the Lie algebra of the Virasoro group that correspond to three types of Poisson pairs obtained this way. In particular, the $\mathrm{CH}$ equation corresponds to the geodesic flow on the diffeomorphism group of the circle. For an alternative approach to this correspondence and a discussion of similar (though leading to a PDE with very different analytic properties) construction for the Bott-Virasoro group we refer the reader to [5] and references therein. It should be noted that within this geometric approach two Poisson structures for the $\mathrm{CH}$ equation are obtained in two different ways. Within our approach employing parametrisation $\left(\Gamma, E_{0}\right)$ of the phase space all these compatible Poisson structures are written in a simple unified way.

It is also worth mentioning that for two special choices of $f(z)=1$ or $f(z)=z$ our analytic formula leads to two specific solutions of the classical Yang-Baxter equation. These rational and trigonometric solutions determine structural constants of a quadratic Poisson algebra [12]. Thus our analytic approach yields Poisson structures traditionally obtained within a group-theoretic framework (see e.g. [18]).

Our formula for Poisson bracket in terms of contour integration of some special differential is a further development of ideas of [9]. In the case $f(z)=1$ it leads to the formula for the Atiyah-Hitchin bracket on Weyl functions, [2, 20].

\section{Analytic Poisson brackets on meromorphic functions.}

In this section we introduce an analytic Poisson bracket and study its properties. We consider our construction in the simplest setting of rational functions on the Riemann sphere. Obvious modifications will be made for the case of Weyl (meromorphic) functions associated with the string spectral problem. 
2.1. Definition of the bracket. We consider the space of pairs $(\mathbb{C P}, \chi)$, where $\mathbb{C P}$ is a Riemann sphere with one marked point and $\chi(z)$ is a rational function of degree $N$. Marked point is called infinity $(\infty)$ and it corresponds to the value $\infty$ of the fixed unformization parameter $z$. The function $\chi(z)$ is a rational function of degree $N$ which vanishes at infinity. Generically, such function can be written in the form

$$
\chi(z)=\frac{\mathcal{S}(z)}{\Delta(z)}=\sum_{k=1}^{N} \chi_{k}(z)=\sum_{k=1}^{N} \frac{\rho_{k}}{z_{k}-z},
$$

where $\mathcal{S}(x)$ is of degree $N-1$ and $\Delta(z)$ is monic of degree $N$ with distinct roots; Rat $_{N}$ is the space of all such rational functions.

Now we are ready to introduce a family of compatible Poisson brackets on the space of pairs. Consider a differential $\omega_{p q}^{f}$ which depends on the entire function $f$ and two points $p$ and $q$ on the curve

$$
\omega_{p q}^{f}=\frac{1}{2 \pi i} \frac{f(z) \chi(z) d z}{(z-p)(z-q)}(\chi(p)-\chi(q)) .
$$

Any point $p \in \mathbb{C P}$ can be viewed as a function on pairs, $p:(\mathbb{C P}, \chi) \rightarrow \chi(p)$.

For any two points $p$ and $q$ which are away from the poles of some $\chi_{0}$ we define the Poisson bracket in a vicinity of the pair $\left(\mathbb{C P}, \chi_{0}\right)$ by the formula

$$
\{\chi(p), \chi(q)\}_{\omega^{f}} \equiv \sum_{m=1}^{N} \int_{\widehat{O}_{m}} \omega_{p q}^{f},
$$

where $O_{m}$ is a small circle enclosing the pole $z_{m}$ and traversed clockwise. Later we will prove that (2.1) defines a genuine Poisson bracket, i.e. it satisfies the Jacobi identity

$$
\{\{\chi(p), \chi(q)\}, \chi(r)\}+\{\{\chi(q), \chi(r)\}, \chi(p)\}+\{\{\chi(r), \chi(p)\}, \chi(q)\}=0,
$$

2.2. Relation to the Yang-Baxter equation. By Cauchy theorem the integral in (2.1) can be computed using residues

$$
\{\chi(p), \chi(q)\}_{\omega^{f}}=\underset{p}{\operatorname{res}} \omega_{p q}^{f}+\underset{q}{\operatorname{res}} \omega_{p q}^{f}+\underset{\infty}{\operatorname{res}} \omega_{p q}^{f} .
$$

There are two important cases when the residue at infinity vanishes.

When $f(z)=1$, we obtain

$$
\{\chi(p), \chi(q)\}_{\omega^{1}}=\frac{(\chi(p)-\chi(q))^{2}}{p-q} .
$$

This formula for the Atiyah-Hitchin bracket, $[2,20]$, is associated with the rational solution of the Yang-Baxter equation. A well-known rational solution of the Yang-Baxter (YB) equation describes a quadratic Poisson algebra, see [12], with two generators $^{3} \mathcal{S}(z)$ and $\Delta(z)$ such that

$$
\begin{aligned}
\{\mathcal{S}(q), \mathcal{S}(p)\}_{\omega^{1}} & =\{\Delta(q), \Delta(p)\}_{\omega^{1}}=0 \\
\{\mathcal{S}(q), \Delta(p)\}_{\omega^{1}} & =\frac{\mathcal{S}(q) \Delta(p)-\mathcal{S}(p) \Delta(q)}{q-p}
\end{aligned}
$$

\footnotetext{
${ }^{3}$ Here $\mathcal{S}(z)$ and $\Delta(z)$ are arbitrary functions of the parameter $z$.
} 
These relations imply $(2.2)$ for the function $\chi(z)=\mathcal{S}(z) / \Delta(z)$. It is interesting to note that relation (2.2) together with (2.3) implies (2.4). Therefore, (2.2) can be viewed as an equivalent form of a rational solution of the YB equation.

In the second case $f(z)=z$, we have

$$
\{\chi(p), \chi(q)\}_{\omega^{z}}=\frac{(p \chi(p)-q \chi(q))}{p-q} \times(\chi(p)-\chi(q)) .
$$

This case is connected with the trigonometric solution of the YB equation. The quadratic algebra associated with this solution is

$$
\begin{aligned}
\{\mathcal{S}(q), \mathcal{S}(p)\}_{\omega^{z}} & =\{\Delta(q), \Delta(p)\}_{\omega^{z}}=0 \\
\{\mathcal{S}(q), \Delta(p)\}_{\omega^{z}} & =\frac{1}{q-p}\left[\frac{q+p}{2} \mathcal{S}(q) \Delta(p)-p \mathcal{S}(p) \Delta(q)\right] .
\end{aligned}
$$

These relations imply (2.5). Relation (2.5) together with (2.6) implies (2.7). Therefore (2.5) is an equivalent way to write a trigonometric solution of the Y-B equation.

In the theory of completely integrable systems the role of functions $\mathcal{S}$ and $\Delta$ is played by the entries of the monodromy matrix of the associated spectral problem, $[10]$.

It is interesting to look at the simplest case $f(z)=z^{2}$ for which the residue at infinity does not vanish. As before we compute

$$
\{\chi(p), \chi(q)\}_{\omega^{z^{2}}}=\frac{\left(p^{2} \chi(p)-q^{2} \chi(q)\right)}{p-q} \times(\chi(p)-\chi(q))+c_{0},
$$

and

$$
\left\{\chi(q), c_{0}\right\}_{\omega^{z^{2}}}=q^{2} \chi^{2}(q)+\left(c_{0} q+c_{1}\right) \chi(q) .
$$

Here $c_{0}, c_{1}$ are the coefficients in the Laurent series $\chi(q)=-\sum_{j=0}^{\infty} c_{j} z^{-j-1}$. We see that quadratic algebra is not closed and it has to be extended by coefficients at infinity.

2.3. Canonical coordinates. Generically, parameters $z_{1}, \ldots, z_{N}$ and $\rho_{1}, \ldots, \rho_{N}$ play the role of coordinates on the space of pairs.

Theorem 2.1. The Poisson bracket (2.1) in $z-\rho$ coordinates has the form

$$
\begin{aligned}
\left\{\rho_{k}, \rho_{n}\right\} & =\frac{\left(f\left(z_{k}\right)+f\left(z_{n}\right)\right) \rho_{k} \rho_{n}}{z_{n}-z_{k}}\left(1-\delta_{k}^{n}\right), \\
\left\{\rho_{k}, z_{n}\right\} & =\rho_{k} f\left(z_{n}\right) \delta_{k}^{n}, \\
\left\{z_{k}, z_{n}\right\} & =0 .
\end{aligned}
$$

Proof. The proof resembles the proof of Theorem 2 in [20], but is somewhat simpler due to a general nature of our approach.

One can represent $\rho$ 's and z's using contour integrals

$$
\rho_{k}=-\frac{1}{2 \pi i} \int_{\overbrace{k}} \chi(\zeta) d \zeta, \quad \rho_{k} z_{k}=-\frac{1}{2 \pi i} \int_{\mathscr{O}_{k}} \zeta \chi(\zeta) d \zeta
$$


Therefore, for $k \neq n$ we have

$$
\begin{aligned}
& \left\{\rho_{k}, \rho_{n}\right\}=\frac{1}{(2 \pi i)^{2}} \int_{\overbrace{O_{k}}} \int_{\overbrace{n}}\{\chi(\zeta), \chi(\eta)\} d \zeta d \eta \\
& =\frac{1}{(2 \pi i)^{2}} \int_{\overbrace{k}} \int_{\overbrace{n}} d \zeta d \eta \frac{1}{2 \pi i} \int_{\sum_{m} \widetilde{O}_{m}} \frac{f(z) \chi(z) d z}{(z-\zeta)(z-\eta)}(\chi(\zeta)-\chi(\eta)) \\
& =\frac{1}{(2 \pi i)^{2}} \int_{\overbrace{O_{k}}} \int_{\overbrace{n}} d \zeta d \eta \frac{1}{2 \pi i} \int_{\widetilde{O}_{O_{m}}} \frac{f(z) \chi(z) d z}{(z-\zeta)(z-\eta)} \chi(\zeta) \\
& -\frac{1}{(2 \pi i)^{2}} \iint d \zeta d \eta \frac{1}{2 \pi i} \int \frac{f(z) \chi(z) d z}{(z-\zeta)(z-\eta)} \chi(\eta) \\
& =A-B \text {. }
\end{aligned}
$$

The contours are given in Figure 2.
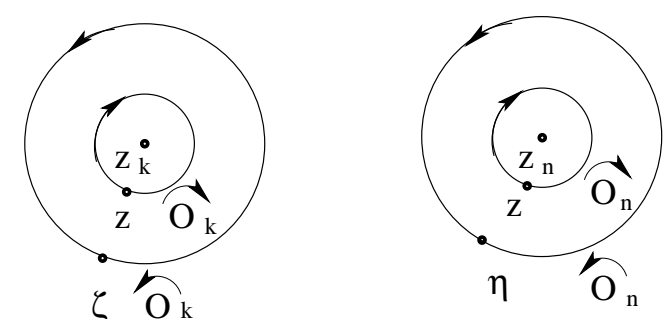

Figure 2. Contours for $k \neq n$.

Using Fubini's theorem

$$
\begin{aligned}
A & =\frac{1}{2 \pi i} \int_{\sum_{m} \widetilde{O}_{m}} d z f(z) \chi(z) \times \frac{1}{2 \pi i} \int_{\overbrace{k}} \frac{\chi(\zeta) d \zeta}{z-\zeta} \times \frac{1}{2 \pi i} \int_{\overbrace{n}} \frac{d \eta}{z-\eta} \\
& =\frac{1}{2 \pi i} \int_{\overbrace{O_{n}}} d z f(z) \chi(z) \times \frac{-\rho_{k}}{z-z_{k}} \times(-1)=\frac{f\left(z_{n}\right) \rho_{n} \rho_{k}}{z_{n}-z_{k}} .
\end{aligned}
$$

Similarly we have

and this implies (2.8).

$$
B=\frac{f\left(z_{k}\right) \rho_{n} \rho_{k}}{z_{k}-z_{n}}
$$

To derive (2.9), for $k \neq n$ we compute

$$
\left\{z_{k} \rho_{k}, \rho_{n}\right\}=\frac{\left(f\left(z_{k}\right)+f\left(z_{n}\right)\right) z_{k} \rho_{k} \rho_{n}}{z_{n}-z_{k}} .
$$

On the other hand, using Leibnitz rule

$$
\left\{z_{k} \rho_{k}, \rho_{n}\right\}=\rho_{k}\left\{z_{k}, \rho_{n}\right\}+z_{k}\left\{\rho_{k}, \rho_{n}\right\} .
$$


This implies $\left\{\rho_{k}, z_{n}\right\}=0$ for $k \neq n$. Derivation is more complicated in the case $k=n$ :

$$
\begin{aligned}
& \left\{z_{n} \rho_{n}, \rho_{n}\right\}=\frac{1}{(2 \pi i)^{2}} \int_{\widehat{O}_{n}} \int_{\widehat{O}_{n}} \zeta\{\chi(\zeta), \chi(\eta)\} d \zeta d \eta \\
& =\frac{1}{(2 \pi i)^{2}} \int_{\widehat{O}_{n}} \int_{\widehat{O}_{n}} \zeta d \zeta d \eta \frac{1}{2 \pi i} \int_{\sum_{m} \widehat{O}_{m}} \frac{f(z) \chi(z) d z}{(z-\zeta)(z-\eta)}(\chi(\zeta)-\chi(\eta)) \\
& =\frac{1}{(2 \pi i)^{2}} \int_{\widehat{O}_{n}} \int_{\widehat{O}_{n}} \zeta d \zeta d \eta \frac{1}{2 \pi i} \int_{\sum_{m} \widehat{O}_{m}} \frac{f(z) \chi(z) d z}{(z-\zeta)(z-\eta)} \chi(\zeta) \\
& -\frac{1}{(2 \pi i)^{2}} \int_{\widetilde{O}_{n}} \int_{\widehat{O}_{n}} \zeta d \zeta d \eta \frac{1}{2 \pi i} \int_{\sum_{m} \widehat{O}_{m}} \frac{f(z) \chi(z) d z}{(z-\zeta)(z-\eta)} \chi(\eta) \\
& =A-B \text {. }
\end{aligned}
$$

The contours are given in Figure 3.

Using Fubini's theorem

$$
\begin{aligned}
A & =\frac{1}{2 \pi i} \int_{\widehat{O}_{n}} d \zeta \zeta \chi(\zeta) \times \frac{1}{2 \pi i} \int \frac{f(z) \chi(z) d z}{z-\zeta} \times \frac{1}{2 \pi i} \int_{\widehat{O}_{n}} \frac{d \eta}{z-\eta} \\
& =\frac{1}{2 \pi i} \int_{\widehat{O}_{m} \widehat{O}_{m}} d \zeta \zeta \chi(\zeta) \times \frac{1}{2 \pi i} \int_{\widehat{O}_{n}} \frac{f(z) \chi(z) d z}{z-\zeta} \times(-1) \\
& =\frac{1}{2 \pi i} \int_{\widehat{O}_{n}} d \zeta\left(\zeta-z_{n}+z_{n}\right) \chi(\zeta) \times \frac{f\left(z_{n}\right) \rho_{n}}{z_{n}-\zeta} \times(-1)
\end{aligned}
$$

$$
=-\rho_{n}^{2} f\left(z_{n}\right)-z_{n} \rho_{n} f\left(z_{n}\right) \frac{1}{2 \pi i} \int_{\widehat{O}_{n}} \frac{\chi(\zeta) d \zeta}{z_{n}-\zeta} .
$$


Similarly,

$$
\begin{aligned}
B & =\frac{1}{2 \pi i} \int_{\widehat{O}_{n}} d \eta \chi(\eta) \times \frac{1}{2 \pi i} \int \frac{f(z) \chi(z) d z}{z-\eta} \times \frac{1}{2 \pi i} \int_{\widehat{O}_{n}} \frac{\zeta d \zeta}{z-\zeta} \\
& =\frac{1}{2 \pi i} \int_{\mathscr{O}_{n}} d \eta \chi(\eta) \times \frac{1}{2 \pi i} \int_{\widetilde{O}_{m}} \frac{f(z) \chi(z) d z}{z-\eta} \times(-z) \\
& =\frac{1}{2 \pi i} \int_{\widehat{O}_{n}} d \eta \chi(\eta) \times \frac{-z_{n} f\left(z_{n}\right) \rho_{n}}{z_{n}-\eta} \\
& =-z_{n} \rho_{n} f\left(z_{n}\right) \frac{1}{2 \pi i} \int_{\widehat{O}_{n}} \frac{\chi(\eta) d \eta}{z_{n}-\eta}
\end{aligned}
$$

Therefore,

and this implies (2.10).

$$
\left\{z_{n} \rho_{n}, \rho_{n}\right\}=-\rho_{n}^{2} f\left(z_{n}\right),
$$

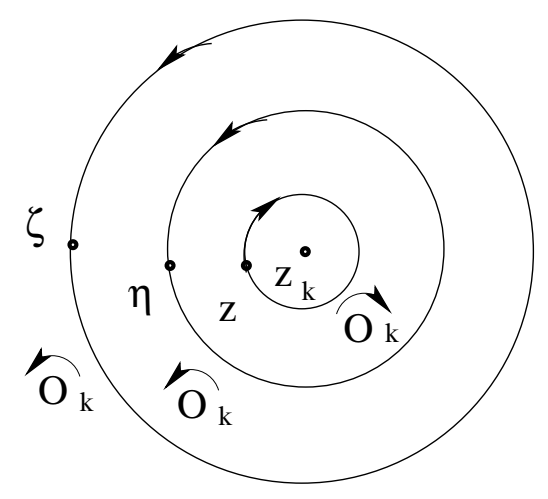

Figure 3. Contours for $k=n$.

To obtain (2.8), for $k \neq n$, as before we compute

$$
\left\{z_{k} \rho_{k}, z_{n} \rho_{n}\right\}=\frac{\left(f\left(z_{k}\right)+f\left(z_{n}\right)\right) z_{k} z_{n} \rho_{k} \rho_{n}}{z_{n}-z_{k}} .
$$

On the other hand,

$$
\left\{z_{k} \rho_{k}, z_{n} \rho_{n}\right\}=z_{k} z_{n}\left\{\rho_{k}, \rho_{n}\right\}+z_{k} \rho_{n}\left\{\rho_{k}, z_{n}\right\}+\rho_{k} z_{n}\left\{z_{k}, \rho_{n}\right\}+\rho_{k} \rho_{n}\left\{z_{k}, z_{n}\right\} .
$$

This together with (2.9) implies (2.10).

One can easily recompute the bracket (2.1) in terms of the coordinates

$$
z_{1}, \ldots, z_{N}, \quad S\left(z_{1}\right), \ldots, S\left(z_{N}\right) .
$$

Namely, using $S\left(z_{k}\right)=-\Delta^{\prime}\left(z_{k}\right) \rho_{k}$ we obtain

$$
\left\{S\left(z_{k}\right), z_{n}\right\}=f\left(z_{n}\right) S\left(z_{k}\right) \delta_{k}^{n},
$$


and all other brackets vanish

$$
\left\{S\left(z_{k}\right), S\left(z_{n}\right)\right\}=\left\{z_{k}, z_{n}\right\}=0 .
$$

Note that this result implies the Jacobi identity for the bracket and compatibility of the brackets with different $f$ 's.

\section{The Liouville correspondence and spectral theory of the string}

3.1. Basic constructions. We consider the Camassa-Holm equation (1.1) in the space $\mathcal{M}$ of all smooth nonnegative functions decaying fast enough at infinity so that (1.3) holds. We also need $\mathcal{M}_{0} \in \mathcal{M}$ - the subspace of functions which vanish far enough to the left. $\mathcal{M}$ and $\mathcal{M}_{0}$ are invariant under the $\mathrm{CH}$ flow, [7].

The $\mathrm{CH}$ equation is a compatibility condition between

$$
D^{2} f-\frac{1}{4} f+\lambda m f=0
$$

and

$$
f^{\bullet}=-\left(v+\frac{1}{2 \lambda}\right) D f+\frac{1}{2}(D v) f ;
$$

i.e., $\left(D^{2} f\right)^{\bullet}=D^{2}\left(f^{\bullet}\right)$ is equivalent to (1.1).

The standard Liouville's transformation

$$
x \rightarrow \xi(x)=2 \tanh x / 2, \quad f(x) \rightarrow f(\xi)=\frac{f(x)}{\cosh x / 2}
$$

converts (3.1) into the string spectral problem

$$
f^{\prime \prime}+\lambda g f=0,
$$

with $g(\xi)=m(x) \cosh ^{4} x / 2$ and $-2 \leq \xi \leq+2$. The transformation changes the length element by the rule

$$
d x \longrightarrow d \xi=d x \mathcal{J}(\xi), \quad \text { with } \quad \mathcal{J}(\xi)=1-\frac{\xi^{2}}{4} .
$$

Under condition (1.3), the string is regular, i.e., its mass is finite:

$$
\int_{-2}^{+2} g(\xi) d \xi=\int_{-\infty}^{+\infty} m(x) \cosh ^{2} x / 2 d x<\infty .
$$

Evidently, for initial data from $\mathcal{M}_{0}$ there is an interval of length $l$ where the potential vanishes: $g(\xi)=0, \quad \xi \in[-2,-2+l]$. The transformation reduces problem (3.1) with two singular ends to the regular string on finite interval. It should be mentioned that a direct approach to the spectral problem (3.1) was initiated in [6] and then developed in [17].

The spectral theory of a string with nonnegative mass was constructed by M.G. Krein in the 1950's and is presented in [14], see also [11, 8].

To formulate the results we need to introduce two solutions $\varphi(\xi, \lambda)$ and $\psi(\xi, \lambda)$ of the eigenvalue problem (3.2) with standard normalization (see (1.6)). The functions $\varphi(2, \lambda)$ and $\psi(2, \lambda)$ can be written in the form

$$
\varphi(2, \lambda)=\prod_{k=1}^{\infty}\left(1-\frac{\lambda}{\mu_{k}}\right), \quad \psi(2, \lambda)=4 \prod_{k=1}^{\infty}\left(1-\frac{\lambda}{\lambda_{k}}\right) .
$$


Their roots interlace each other

$$
0<\mu_{1}<\lambda_{1}<\mu_{2}<\lambda_{2}<\ldots
$$

For the string $\mathcal{S}_{0}$ with fixed left and right ends according to the general theory

$$
E_{0}(\lambda)=-\frac{\varphi(2, \lambda)}{\psi(2, \lambda)}=-\frac{1}{4}+\sum_{k=1}^{\infty}\left(\frac{1}{\lambda_{k}-\lambda}-\frac{1}{\lambda_{k}}\right) \rho_{k} .
$$

The poles of $E_{0}(\lambda)$ are zeros of $\psi(2, \lambda)$ and they do not move under the $\mathrm{CH}$ flow. Introducing

$$
z_{k}=-\frac{1}{\lambda_{k}}, \quad \rho_{k}^{\prime}=\frac{\rho_{k}}{\lambda_{k}^{2}}
$$

we have the identity

$$
\frac{\rho_{k}}{\lambda_{k}-\lambda}=\frac{\rho_{k}^{\prime}}{z_{k}-z}+\frac{\rho_{k}}{\lambda_{k}}
$$

Therefore for the function $E_{0}$ we obtain

$$
E_{0}(z)=-\frac{1}{4}+\sum_{k=1}^{\infty} \frac{\rho_{k}^{\prime}}{z_{k}-z}
$$

In this form it is suitable for our purposes.

3.2. The first and the second analytic Poisson brackets for the Weyl function. Very little have to be added now in order to introduce a family of compatible Poisson brackets. The pair $\left(\Gamma, E_{0}\right)$ provides a parametrization of the phase space $\mathcal{M}$, see [19]. We will write Poisson brackets in terms of the pair.

Consider a differential $\omega_{p q}^{f}$ on the lower component of the spectral curve $\Gamma_{-}$which depends on the entire function $f$ and two points $p$ and $q$

$$
\omega_{p q}^{f}=\frac{1}{2 \pi i} \frac{f(z) E_{0}(z) d z}{(z-p)(z-q)}\left(E_{0}(p)-E_{0}(q)\right) .
$$

Any point $p \in \Gamma_{-}$can be considered as a function on pairs $p:\left(\Gamma, E_{0}\right) \rightarrow E_{0}(p)$. For any two points $p$ and $q$ which are away from the poles of some $E_{0}$ we define the Poisson bracket in a vicinity of the pair $\left(\Gamma, E_{0}\right)$ by the formula

$$
\left\{E_{0}(p), E_{0}(q)\right\}_{\omega f}=\sum_{m} \int_{\tilde{O}_{m}} \omega_{p q}^{f},
$$

where the circles $O_{m}$ are traversed clockwise and surround points $z_{m}$. The sum over all small contours converges since its may be replaced by one integral over a large contour surrounding all poles $z_{k}$.

Now we need the following

Theorem 3.1. [19]. Let $N(\zeta)=N_{0}$ be a real constant, possibly infinity. Then,

$$
\left\{E_{N_{0}}(\lambda), E_{N_{0}}(\mu)\right\}_{J_{0}}=\frac{\lambda \mu}{\lambda-\mu}\left(E_{N_{0}}(\lambda)-E_{N_{0}}(\mu)\right)^{2} .
$$


If one changes the spectral parameter by the rule

$$
p=-\frac{1}{\lambda}, \quad q=-\frac{1}{\mu},
$$

then the formula (3.9) becomes

$$
\left\{E_{N_{0}}(p), E_{N_{0}}(q)\right\}_{J_{0}}=\frac{\left(E_{N_{0}}(p)-E_{N_{0}}(q)\right)^{2}}{p-q} .
$$

Therefore, comparing this with (2.2) we see that the Poisson bracket $J_{0}=m D+D m$ corresponds to the choice $f(z)=1$ in the formula (3.8).

It can be proved either by similar direct calculation or by using the recurrence relation (1.5) that the second Poisson bracket $J_{1}=D-D^{3}$ corresponds to the choice $f(z)=z$ in $(3.8)$.

The formula (3.8) defines the Poisson bracket for any choice of an entire function $f(z)$, though its explicit expression in terms of the phase space $\mathcal{M}$ is not known to us. It is not even known when the corresponding Poisson tensor on $\mathcal{M}$ is local.

\section{References}

[1] V.I. Arnold, Mathematical Methods of Mechanics. Graduate Texts in Mathematics, 60. Springer-Verlag, New York, 2000. xvi+516 pp.

[2] M. Atiyah and N. Hitchin, The Geometry and Dynamics of Magnetic Monopoles. Princeton Univ. Press, (1988).

[3] R. Camassa, D. Holm and J. Hyman, A new integrable shallow water equation. Advances in Applied Mechanics, 31 (1994), 1-33.

[4] R. Camassa and D. Holm, A completely integrable dispersive shallow water equation with peaked solitons. Phys. Rev. Lett. 71 (1993), 1661-1664.

[5] A. Constantin and B. Kolev, Integrability of invariant metrics on the diffeomorphism group of the circle, J. Nonlinear Sci. 16 (2006), no. 2, 109-122.

[6] A. Constantin and H. P. McKean, A shallow water equation on the circle, Comm. Pure Appl. Math. 52 (1999), no. 8, 949-982.

[7] A. Constantin and L. Molinet, Global weak solutions for a shallow water equation. CMP, 211 (2000) 45-61.

[8] H. Dym and H. McKean, Gaussian Processes, Function Theory, and the Inverse Spectral Problem. Academic Press, New York, Sun Francisco, London. (1976).

[9] L. Faybusovich and M. Gehtman, Poisson brackets on rational functions and multiHamiltonian structures for integrable lattices. Phys. Lett. A, 272 (2000), 236-244.

[10] L. Faddeev and L. Takhtadjian, Hamiltonian methods in the theory of solitons Springer Series in Soviet Mathematics. Springer-Verlag, Berlin, 1987. x+592 pp.

[11] F.R. Gantmacher and M.G. Krein, Oscillation Matrices and Small Oscillations of Mechanical Systems. (Russian) Gostekhizdat, Moscow-Leningrad. (1941).

[12] A. S. Fokas and I.M. Gelfand, Quadratic Poisson algebras and their infinite-dimensional extensions. J. Math Phys. 35 (1994), no 6. June 1994.

[13] I.S. Kac and M.G. Krein, r-functions analytic functions mapping the upper half-plane into itself. Transl. Amer. Math. Soc. 103 (1974), 1-18.

[14] _ On the spectral function of the string. Transl. Amer. Math. Soc. 103 (1974), 19-102.

[15] I.M. Krichever and K.L. Vaninsky, The periodic and open Toda lattice. "Mirror symmetry. IV" AMS/IP Studies in Advance Mathematics, 33 E.D'Hoker, D. Phong, S.T. Yau eds. (2002).

[16] B. Khesin and G. Misiolek, Euler equations on homogeneous spaces and Virasoro orbits Adv. Math. 176 (2003), no. 1, 116-144.

[17] H. P. McKean, Fredholm determinants and the Camassa-Holm hierarchy, Comm. Pure Appl. Math. 56 (2003), no. 5, 638-680.

[18] M. A. Olshanetsky, A. M. Perelomov, A. G. Reyman and M. A. Semenov-Tian-Shansky, Integrable systems. II. Dynamical systems. VII. Encycl. Math. Sci. 16, 83-259. 
[19] K.L. Vaninsky, Equations of Camassa-Holm type and Jacobi ellipsoidal coordinates. Comm Pure and Apllied Math, 46 (2006), 283-307.

[20] _ The Atiyah-Hitchin bracket and open Toda lattice. Journal of Geometry and Physics, 46 (2003), 283-307.

[21] H. Weyl, Uber gewöhnliche Differentialgleichungen mit Singularitäten und die zugehörigen Entwicklungen willkürlichen Funktionen. Math. Ann. 68 (1910), 220-269.

Department of Mathematics, University of Notre Dame, Notre Dame, IN 46556

E-mail address: mgekhtma@nd.edu

Department of Mathematics, Michigan State University, East Lansing, Mi 48824

E-mail address: vaninsky@math.msu.edu 